\title{
Uso de Cadeias de Markov para Otimizar o Sensoriamento Colaborativo do Espectro em Redes 5G
}

\author{
Gabriel C. Ferreira ${ }^{1}$, Priscila Solís Barreto ${ }^{1}$, Marcos F. Caetano ${ }^{1}$, \\ Geraldo P. Rocha Filho ${ }^{1}$, Johanna Vartiainen ${ }^{2}$ e \\ Heikki Karvonen ${ }^{2}$
}

${ }^{1}$ Departamento de Ciência da Computação - Universidade de Brasília (UnB) Caixa Postal 4466 CEP 70910-900 - Brasília - DF - Brasil

gabrielcarvfer@aluno.unb.br, \{pris,mfcaetano, geraldof\}@unb.br

${ }^{2}$ Centre for Wireless Communications - University of Oulu, Oulu, Finland

\{johanna.vartiainen, heikki.karvonen\}@oulu.fi

\begin{abstract}
Cognitive radios are considered critical for better spectrum use in $5 G$ networks. Spectrum sensing is a technology that allows channel status to be determined on a decentralized basis. Collaborative spectrum sensing improves detection by collecting more data from different users in different places, increasing the amount of information available to making decisions. This paper presents a Markov chain-based technique that improves spectrum detection and decreases the number of sensing notifications. Thus, the technique improves the result of classic fusion algorithms for co-operative sensing in the case of wellbehaved access. There was a reduction of up to $\sim 99.67 \%$ in the false alarm rate and up to $\sim 98 \%$ of the associated control traffic, preserving the false negative rate below the tolerable limit of 10\%, mitigating the interference to a channel user.
\end{abstract}

Resumo. Os rádios cognitivos são considerados fundamentais para melhorar o uso do espectro em redes 5G. O sensoriamento de espectro é uma tecnologia que permite que o estado do canal seja determinado de forma descentralizada. O sensoriamento colaborativo do espectro melhora a detecção ao coletar mais dados de diferentes usuários, em diferentes lugares, aumentando a quantidade de informação disponível para a toma de decisões. Este artigo apresenta uma técnica baseada em cadeias de Markov que melhora a detecção do espectro e diminui o número de notificações de sensoriamento. Dessa forma, a técnica melhora o resultado dos algoritmos de fusão clássicos para sensoriamento colaborativo no caso de acesso bem comportado. Observou-se uma redução de até $\sim 99.67 \%$ da taxa de alarmes falsos e até $\sim 98 \%$ do tráfego de controle associado, preservando a taxa de falsos negativos abaixo do limite tolerável de $10 \%$, minimizando interferência ao usuário do canal.

\section{Introdução}

O espectro de radiofrequência (Radio Frequency (RF)) é um recurso escasso, definido como um bem público e gerenciado por agências reguladoras. A administração do espectro é realizada em conjunto pela UIT (União Internacional de Telecomunicações) no 
âmbito global e por órgãos reguladores próprios de cada país ou região. O uso simultâneo do espectro em uma mesma área, na transmissão de vários sinais em uma mesma faixa de frequências, resulta em interferência, o que pode distorcer ou impossibilitar a recepção da informação transmitida. As agências reguladoras outorgam permissões a usuários, chamados Usuário Primário (Primary User) s (PUs), e operadoras para transmitirem em frequências determinadas em regiões específicas. A administração do espectro cria desafios para a implantação de novas tecnologias dado que o recurso é escasso, as tecnologias de comunicação são dinâmicas e muitas das bandas já reservadas podem ser subutilizadas nos domínios de tempo e frequência[3GPP 2009].

A UIT definiu as bandas de $450 \mathrm{MHz}$ a $6 \mathrm{GHz}$ para o IMT (International Mobile Telecommunications) [3GPP 2009] e na linha de evolução em direção às redes móveis de Quinta Geração (5G). Os cenários de Enhanced Mobile Communications (EMC), Massive Machine Type Communications (MMTC) e Ultra Reliable Low Latency Communications (URLLC) [Maternia et al. 2016] podem utilizar qualquer destas bandas, além das bandas candidatas na faixa de $24,25 \mathrm{GHz}$ e $86 \mathrm{GHz}$ [Union 2015]. Em regiões árticas e recentemente no Brasil, um cenário que vem despertando interesse pela comunidade acadêmica é o de acesso a áreas remotas. Nesse contexto, um grande raio de cobertura é o principal requisito para viabilizar as aplicações típicas de 5G nessas áreas [Pirinen et al. 2019].

No cenário de $5 \mathrm{G}$ em áreas remotas ou rurais, observa-se a subutilização nas bandas destinadas à transmissão de sinais de televisão, tais como Very High Frequency (VHF) e Ultra High Frequency (UHF)], conhecidas como TV White Spaces (TVWS) [Sum 2013]. O conceito de Cognitive Radio (CR) tem sido amplamente explorado na literatura [Akyildiz et al. 2011, Tan and Le 2014] como uma tecnologia importante para mitigar o problema da escassez de espectro. O uso de CR permite que usuários sem licença para uso do canal, chamados de Usuário Secundário (Secondary User) s (SUs), acessem oportunisticamente canais subutilizados. Salienta-se, entretanto, que a maneira de alocar o espectro não é uma tarefa trivial, uma vez que o SU não deve interferir na transmissão do PU. Para resolver esse problema, o CR deve detectar o meio de agendar a transmissão durante os tempos ociosos do PU de uma maneira eficaz, ao mesmo tempo em que fornece alta confiabilidade e Qualidade de Serviço (QoS) para os diversos SU, em conjunto com robustez, flexibilidade e ampla cobertura. Uma forma de abordar o problema no âmbito do CR é integrar à camada de Controle de Acesso ao Meio (Medium Access Control) (MAC), técnicas de sensoriamento colaborativo em conjunto com alocação dinâmica do espectro e métodos de escalonamento de recursos [Yau et al. 2018].

$\mathrm{Na}$ literatura, algumas pesquisas abordam o sensoriamento do espectro como uma função localizada em um nó individual [Tan and Le 2014, Liu et al. 2014]. Outros trabalhos exploram as tendências futuras de compartilhamento do espectro para redes $5 \mathrm{G}$ com base em rádios cognitivos[Hu et al. 2018]. No caso do Sensoriamento Colaborativo do Espectro (Collaborative Spectrum Sensing) (CSS), entende-se que é um sistema que inclui uma função de detecção operando em diferentes nós e um centro de fusão centralizado que consolida relatórios diferentes de vários nós e produz uma decisão global sobre a disponibilidade de um determinado canal[Wang et al. 2019, Yau et al. 2018]. Para o CSS em redes sem fio, alguns trabalhos exploram métodos de fusão [Teguig et al. 2012], outros avaliam o consumo de energia [Cichoń et al. 2016] e outros apresentam técnicas de fusão mais eficientes [Fu et al. 2018]. Para redes 5G existem poucas abordagens que 
analisem no CSS a aplicabilidade de modelos matemáticos no processamento do sensoriamento individual, para melhorar a eficiência da rede e aumentar a acurácia da detecção.

Neste trabalho é proposto um novo mecanismo com base em cadeias de Markov para aumentar a acurácia e eficiência na detecção de canais livres em uma rede 5G para áreas remotas. $\mathrm{O}$ mecanismo foi desenvolvido como um parte integrante de uma camada MAC cognitiva que utiliza CSS para acesso oportunístico de TVWS. Foi conduzida uma avaliação da proposta por meio de simulações. Os resultados de simulação mostram que a técnica proposta apresenta uma melhor acurácia em identificar canais disponíveis, assim como uma melhor eficiência da rede ao diminuir significativamente o tráfego de controle conforme o número de nós aumenta. O mecanismo proposto diferencia-se de outros trabalhos pelas seguintes contribuições científicas: a) formulação de uma nova técnica do tipo hard-combining para aumentar a acurácia no resultado do sensoriamento do espectro; b) redução da quantidade de dados de controle transmitidos na rede para a fusão de resultados do sensoriamento colaborativo e (c) avaliação da técnica proposta em novos cenários e novas ferramentas de simulação para redes 5G.

O artigo está organizado da forma seguinte: a Seção 2 apresenta a fundamentação teórica e trabalhos relacionados, a Seção 3 discute o método proposto e seus fundamentos, a Seção 4 apresenta os resultados experimentais e sua análise, e finalmente, a seção 5 contém as conclusões e trabalhos futuros desta pesquisa.

\section{Fundamentação Teórica a e Trabalhos Relacionados}

\subsection{Rádios Cognitivos e Acesso Oportunístico ao Espectro}

Grande parte dos blocos de espectro licenciados são subutilizados longe dos grandes centros urbanos, em especial as bandas de telefonia móvel, rádio FM, televisão analógica e digital [Valenta et al. 2009, Beckman et al. 2010, Hu et al. 2018]. O melhor aproveitamento no uso de bandas licenciadas subutilizadas permite que aplicações específicas usem mais banda, o que aumenta a capacidade da rede e reduz os custos de licenciamento.

Devido a escassez do espectro, as agências reguladoras trabalham em propostas de licenças mais flexíveis que permitam o compartilhamento das bandas licenciadas entre diferentes usuários. Os principais modelos de licenças estudadas são License Shared Access (LSA) e General Authorized Access (GAA). As licenças LSA funcionam mediante autorização por fatias de tempo [Basnet et al. 2017]. Já as licenças GAA funcionam como uma mistura do LSA e Dynamic Spectrum Access (DSA), que permite a priorização de usuários licenciados de serviços protegidos, usuários licenciados de serviços não protegidos e usuários não licenciados.

O rádio cognitivo é um sistema em que a interface de rádio capaz de sensorear seu ambiente, podendo utilizar tecnologias adicionais para obter informações. A partir destas informações, os parâmetros e protocolos operacionais do rádio é adaptado seguindo políticas definidas pelo usuário [Akyildiz et al. 2011, Wang et al. 2019]. O principal método para obter essas informações é o sensoriamento do espectro, complementado por informações de localização e uso do banco de dados geográfico. O banco de dados geográfico pode fornecer informações da ocupação dos canais e é vital para proteger os usuários históricos e melhorar a coexistência com redes secundárias. O conhecimento adquirido permite a reconfiguração do rádio para alcançar objetivos da transmissão, alterando parâmetros tais como potência de transmissão, faixa de frequência, protocolos, 
modulação, entre outros. Dessa forma, são identificadas e aproveitadas as características de transmissão no espectro.

\subsection{Sensoriamento do Espectro}

O sensoriamento do espectro consiste em medir um canal por um período e determinar se existem ou não transmissões, seja pelo nível de energia transmitido, padrões de forma de onda ou padrões de uso das frequências [Yucek and Arslan 2009, Akyildiz et al. 2011]. Os principais objetivos do sensoriamento do espectro são: identificar bandas ociosas e bandas ocupadas para evitar colisões e interferência; identificar aplicações que usam certas bandas o que permite proteção e priorização de usuários e serviços essenciais.

Os métodos de detecção do espectro podem ser divididos de várias maneiras. Uma delas é classificar o método de detecção como cego, semi-cego ou clássico (não cego). A detecção cega não precisa de nenhuma informação sobre o sinal ou ruído. Nessa categoria, uma das técnicas mais simples é o sensoriamento do nível de energia de uma portadora em um canal, usada no Carrier Sense Multiple Access (CSMA) e variantes [Shafiq et al. 2019]. Outras formas de sensoriamento baseadas em energia, correlação temporal ou de forma de onda, ou mesmo filtragem de padrões também podem ser utilizadas [Yucek and Arslan 2009, Akyildiz et al. 2011]. Os métodos semi-cegos requerem informações sobre a potência ou variação do ruído e os métodos de detecção clássicos, como por exemplo a detecção de recurso cicloestacionário, precisam das informações sobre o sinal e/ou a potência do ruído. Ainda, é possível agrupar os métodos de detecção de espectro individual, que abrange técnicas que operam de forma independente, e híbrida, que inclui métodos de dois estágios e métodos cooperativos.

Nos métodos cooperativos, a detecção pode ser realizada de maneira distribuída ou centralizada. Na detecção distribuída, os nós operam independentemente e se comunicam entre si. Os resultados de detecção local são compartilhados com outros nós e as decisões são tomadas localmente. Nesse caso, os custos são mais baixos do que na cooperação centralizada, porque não há necessidade de um Canal de Controle Comum (CCC). Na cooperação centralizada, o processo de detecção é coordenado por um centro de fusão (Fusion Center (FC)), de modo que a decisão final de detecção é entregue a todos os nós cooperativos.

Os relatórios de detecção e sua combinação utilizam uma das técnicas seguintes: Soft, Hard e Quantized combining. As três técnicas diferem quanto a quantidade de bits transmitidos por SU para indicar a presença de um PU, com Hard e Quantized usando 1 bit cada, enquanto Soft usa n bits para representar a probabilidade da detecção [Teguig et al. 2012, Alvi et al. 2014, Fu et al. 2018]. Quanto maior a quantidade de bits, maior a granularidade da informação, o que permite que o algoritmo de fusão utilizado faça uma decisão melhor informada. Apesar disto, o custo de transmissão dos sensoriamentos pode ser proibitivo, dependendo do número de Equipamento de Usuário (User Equipment) s (UEs), número de canais sensoreados e banda disponível. Tipicamente, o tipo de fusão Hard é escolhido pela simplicidade e pelo pequeno overhead. As técnicas mais simples de fusão são baseadas em contagem, como: $O U$ lógico, em que qualquer relatório que aponte a presença do PU é tomado como verdadeiro; $E$ lógico, que define o PU presente se todos os relatórios recebidos indicarem a sua detecção; limiar $K$ de $N$, em que $K$ relatórios positivos dentre $N$ recebidos indicam a presença do PU. 
No CSS convencional grande parte dos nós fazem a detecção que enviam a um elemento central, que decide com um algoritmo de fusão a ocupação do canal. Esse processo consume recursos da rede e aumenta o consumo de energia dos nós. Para minimizar o custo da rede e maximizar o ganho do processo de detecção, uma abordagem é reduzir o número de notificações de nós que pouco contribuem para o algoritmo de fusão. Entretanto, em algumas topologias, a definição dos nós que devem notificar o sensoriamento pode ser um processo não trivial. Por outro lado, a colaboração permite que uma maior quantidade de conhecimento sobre o estado do canal seja agregada, caso os nós estejam suficientemente distantes uns dos outros, produzindo assim um resultado mais acurado da detecção.

Para avaliar a qualidade do CSS são utilizadas métricas tais como Probabilidade de Detecção $\left(P_{d}\right)$, Probabilidade de Falso Negativo $\left(P_{f n}\right)$ e Probabilidade de Alarme Falso (Probability of False Alarm) ( $\left.P_{f a}\right)$ [Fu et al. 2018]. Também pode ser avaliada a quantidade de bits transmitidos pelos dispositivos sensores e o atraso entre o sensoriamento e a atuação quanto ao acesso do canal [Liu et al. 2014].

\subsection{Trabalhos Relacionados}

As cadeias de Markov têm sido utilizadas em propostas que aplicam CR no protocolo Carrier Sense Multiple Access-Collision Avoidance (CSMA/CA) [Wang et al. 2010, Foukalas et al. 2013], em que o controle de acesso ao meio é distribuído e depende exclusivamente do sensoriamento individual dos SUs da rede. Os resultados do sensoriamento são utilizados para controlar o tempo de backoff em uma cadeia de Markov, requerendo certo nível de certeza antes de transmitir. Diferente deste trabalho, a proposta não aborda sensoriamento colaborativo, o que pode resultar no aumento da probabilidade de causar interferência ao PU e tendência à monopolização do canal.

Outras abordagens para o CSMA/CA são propostas em [Li et al. 2012, Tan and Le 2014], em que cada SU da rede faz o sensoriamento em um dado conjunto de canais e compartilha seus resultados com outros nós da rede após o fim deste sensoriamento através de mensagens de broadcast. Em seguida, o CSMA/CA é utilizado para definir qual SU transmitirá em cada canal livre. Entretanto, as propostas apresentadas diferenciam-se desta pesquisa pois os nós não possuem garantias de conhecimento completo e não são sincronizados. Além disto, as abordagens não utilizam nenhuma técnica de melhoria da confiabilidade das amostras e fazem broadcast de seus resultados de sensoriamento em múltiplos canais, aumentando ainda mais o overhead do sensoriamento.

A identificação de nós com melhor capacidade de detecção é fundamental para a eficiência do CSS. Encontrar uma relação eficiente entre o custo e desempenho da detecção, e a quantidade de dados transmitidos no canal comum de controle é complexo. Existem várias propostas para lidar com esse problema. Uma forma é definir que nós com as maiores probabilidades de detecção tenham o melhor desempenho de detecção [Khan et al. 2011]. Também é possível selecionar usuários que possuam uma medida mínima de correlação entre eles [5G-Range 2018] ou selecionar dispositivos com o maior ganho para coletar e encaminhar relatórios dos outros nós para o FC [Cichoń et al. 2016]. Em [Malady and da Silva 2008], os autores estudam métodos de agrupamento (aleatórios, baseados em referência, estatísticos e baseados em distância) para selecionar usuários adequados. Desses, apenas o método de agrupamento aleatório não precisa saber as 
informações de localização. Em [Wang et al. 2015], os nós são selecionados com base na potência do sinal do PU recebido e na distância do estação radio base.

A pesquisa bibliográfica mostra que é inédita uma proposta que utilize um modelo de cadeias de Markov no CSS nos cenários de redes 5G para áreas remotas, que tenha como objetivo aumentar a acurácia da detecção e diminuir o volume de dados transmitidos no canal de controle. Por isso, a seguir será apresentada a proposta de sensoriamento com base nas cadeias de Markov.

\section{Proposta de Sensoriamento com Cadeias de Markov}

Nesta seção é apresentado um mecanismo para filtragem de ruído no sensoriamento individual do tipo hard-combining com base em cadeias de Markov. O mecanismo proposto tem como objetivo aumentar a acurácia do sensoriamento colaborativo ao reduzir alarmes falsos e falsos negativos, estes últimos de vital importância, dado que valores altos indicam potencial interferência ao PU. Ainda, o mecanismo tem como finalidade melhorar a eficiência entre o custo e o desempenho da detecção ao diminuir a quantidade de dados transmitidos no canal de controle comum da rede. Para um melhor entendimento do mecanismo proposto, são necessárias duas etapas:(i) fundamentos e premissas, Subseção 3.1 e (ii) comportamento do modelo, Subseção 3.2.

\subsection{Fundamentos e Premissas}

O procedimento comum de Sensoriamento do Espectro (SS) tem como base um ciclo de espera, sensoriamento e transmissão de resultados, ilustrado na Figura 1(a). A esse ciclo devem ser acrescidas duas checagens para implementar uma cadeia de Markov de cinco estados. O mecanismo resultante de SS é apresentado na Figura 1(b). Para cada ciclo de execução, caso o resultado do sensoriamento atual ( $\left.R_{\text {sense }}\right)$ seja equivalente ao do passado $\left(R_{\text {prev }}\right)$, acumula-se a incerteza de eventos idênticos acontecerem consecutivamente $\left(P_{\text {acum }}\right)$. Por definir que o método é do tipo hard-combining, os resultados do sensoriamento são binários o acúmulo de probabilidade é feito em saltos de $\frac{1-P_{\text {acum }}}{2}$, equivalentes ao lançamento de uma moeda. Quando esta incerteza $P_{\text {acum }}$ atinge um dado limiar, por exemplo $90 \%$, assume-se que o resultado do sensoriamento $R_{\text {sense }}$ é correto visto que é improvável de acontecer em um cenário em que um PU de fato não esteja transmitindo, e assim, armazena-se o valor a ser reportado em $R_{\text {markov }}$. Caso o limiar $P_{\text {acum }}$ não tenha sido ultrapassado, o UE não reporta o resultado do sensoriamento atual, e a célula de telefonia Evolved Node-B (eNB) assume que o resultado reportado no passado continua a ser válido.

As premissas utilizadas para definir o mecanismo proposto dentro do contexto de CSS são as seguintes:

P.1 o SS individual depende única e exclusivamente da capacidade do próprio dispositivo em detectar a utilização de um dado canal;

P.2 existe uma curva de probabilidades de detecção $p_{d}(d)$ dependente da distância $d$ entre o dispositivo sensor e a fonte de interferência, derivada de medições ou simulação na camada de enlace de um algoritmo padrão de SS;

P.3 a probabilidade de alarmes falsos $p_{f a}$ do algoritmo de SS é conhecida, também derivada de medições ou simulação na camada de enlace;

P.4 ambos (UEs) e (PUs) estão com micromobilidade; 


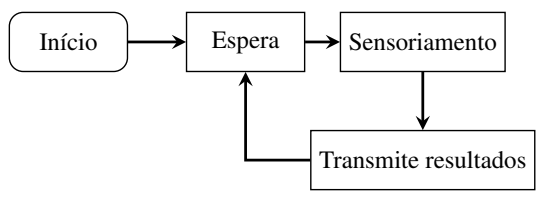

(a) Passos do Sensoriamento Individual

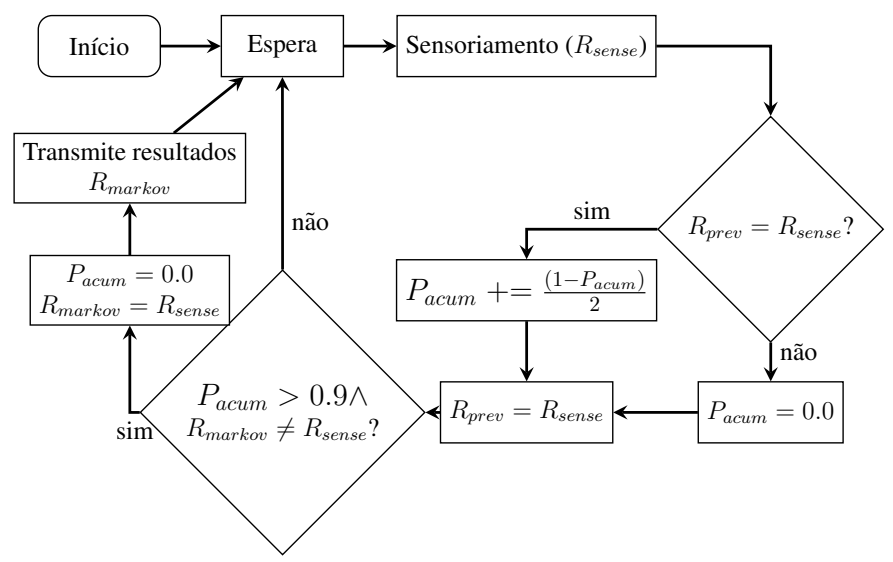

(b) Sensoriamento Individual com Cadeia de Markov

Figura 1. Fluxograma do ciclo de sensoriamento do espectro individual

P.5 $p_{d}>>1-p_{d}$, caso o PU esteja transmitindo;

P.6 $p_{f a}<<1-p_{f a}$, caso o PU não esteja transmitindo.

\subsection{Comportamento do Modelo}

Em relação ao sensoriamento do espectro, ao partir das premissas P.2 e P.3, a probabilidade do sensoriamento dar um resultado positivo, seja ele verdadeiro ou falso, é calculada pela Equação 1. O valor $d$ indica a distância entre o UE e o PU, enquanto $p u_{\text {transmitindo }}$ indica se o PU está transmitindo em um dado momento $t$. Se o dispositivo é estático, conforme a premissa P.4, calcula-se $d(t)=d(0) \forall t \in[0, \infty)$. A distribuição de $n$ amostras com $k$ sensoriamentos de um UE indicando a presença de um PU é calculada pela Equação 2.

$$
\begin{gathered}
p_{\text {sense }}\left(t, d, p u_{\text {transmitindo }}\right)= \begin{cases}p_{d}(d(t)), & \text { se } p u_{\text {transmitindo }}(t)=1 . \\
p_{f a}, & \text { caso contrário. }\end{cases} \\
\left(\begin{array}{l}
n \\
k
\end{array}\right) p_{\text {sense }}^{k}\left(1-p_{\text {sense }}\right)^{n-k}
\end{gathered}
$$

Caso as premissas P.5 e P.6 sejam verdadeiras, a probabilidade de $k$ em $n$ amostras serem positivas é próxima de $p_{\text {sense }}$ se $k \rightarrow n$ para um $n$ pequeno. Se $p_{\text {sense }} \Leftarrow$ $\left(1-p_{\text {sense }}\right)$, a probabilidade das $k$ em $n$ amostras se afasta de $p_{\text {sense }}$ conforme $n$ cresce. Deste mesmo comportamento, se observa o efeito colateral na curva de probabilidade de detecção $P_{d}(d)$ apresentada na Figura 2(a), em que a probabilidade de detecção simulada (quadrados em azul) é atenuada (triângulos em verde) quando $N$ aumenta.

A agregação dos resultados segue a cadeia de Markov de $Z$ estados mostrada na Figura 2(b), representando a contagem de eventos consecutivos com um mesmo resultado $(B-S$ e $T-Z)$, seja o resultado positivo $(P)$ ou negativo $(1-P)$. Quanto o resultado é diverso do anterior, a certeza acumulada $C_{\text {acum }}=1-P^{K}$ ou $C_{a c u m}=1-(1-P)^{W}$ pelos $K=W$ eventos consecutivos é perdida e a contagem recomeça. Tendo em vista um dado limiar $L$ de certeza acumulada e uma probabilidade $P$, calcula-se o número de estados $Z$ da cadeia de Markov desejada. Baseado no lançamento de uma moeda, $P=1-P=0.5$, 


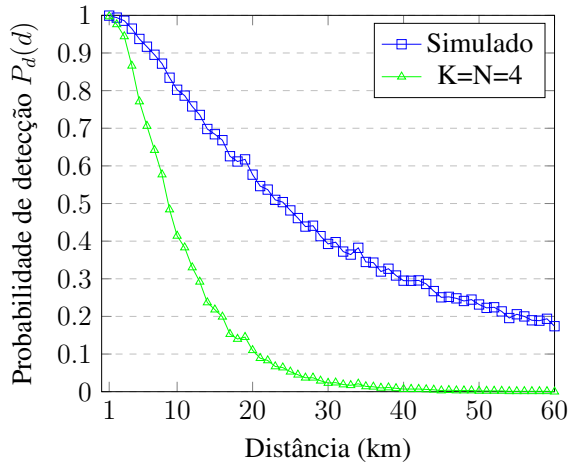

(a) Curva de probabilidade de detecção com base na distância [5G-Range 2018]

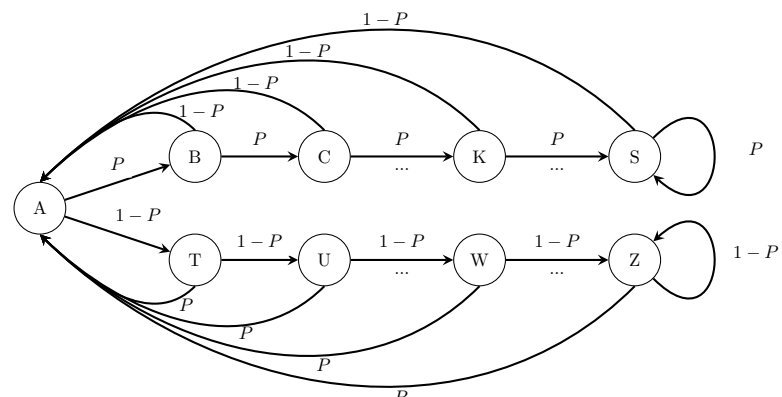

(b) Cadeia de Markov com $Z$ estados

Figura 2. Curvas de probabilidade de detecção e cadeia de Markov proposta

e um limiar de certeza de $L=0.9$, temos que o número de estados da cadeia de Markov é $Z=|(A, . ., S)|+|(T, . ., Z)|=5+4=9$, com $\left(\frac{Z-1}{2}\right)=4$ transições de $A$ até $S$ e $Z$, equivalente a atenuação da distribuição binomial com $K=N=4$ quando o UE está há 10km de distância de um PU, conforme mostrado (triângulos em verde) na Figura 2(a). A escolha do limiar de certeza calibra a troca de certeza do resultado, conjuntamente com atraso entre o início da transmissão do PU e a detecção do mesmo, e o alcance da detecção.

A quantidade de dados com resultados dos sensoriamentos cresce com o aumento do número de dispositivos sensores (UEs), aumentando a carga da rede. A não retransmissão de resultados idênticos consecutivos é um mecanismo simples para a redução de transmissões, onde o nó centralizador (eNB) assume que o estado do canal se manteve inalterado. O efeito suavizador deste mecanismo pode ser visto na Figura 3, em que existem 10 transições de estado no sensoriamento padrão, contra um total de 31 sensoriamentos, reduzindo aproximadamente $\frac{2}{3}$ das transmissões. A cadeia de Markov, porém, reduz ainda mais as transições de estado, suavizando os resultados do sensoriamento. No exemplo da Figura 3, o sensoriamento com a cadeia de Markov tem apenas uma única transição de estado, vide que não houveram sensoriamentos consecutivos que apontassem para a não presença do PU em função do acúmulo de certeza acumulada.

\section{Resultados de Simulação}

Esta seção avalia o desempenho do mecanismo proposto. Para isso, a seguir será apresentado o ambiente de simulação, as métricas utilizadas e os resultados obtidos.

\subsection{Ambiente de Simulação}

O mecanismo foi implementado no simulador NS-3 LENA [Baldo et al. 2011]. Foram realizadas várias extensões no simulador para adaptar a camada MAC [Ferreira et al. 2019] e para implementar o ciclo descrito na Figura 1(b). Ainda, foram integrados ao simulador os resultados de sensoriamento individual do espectro produzidos no MATLAB pelo algoritmo WIBA [Vartiainen et al. 2019, Saarnisaari and Vartiainen 2018], no padrão apre- 


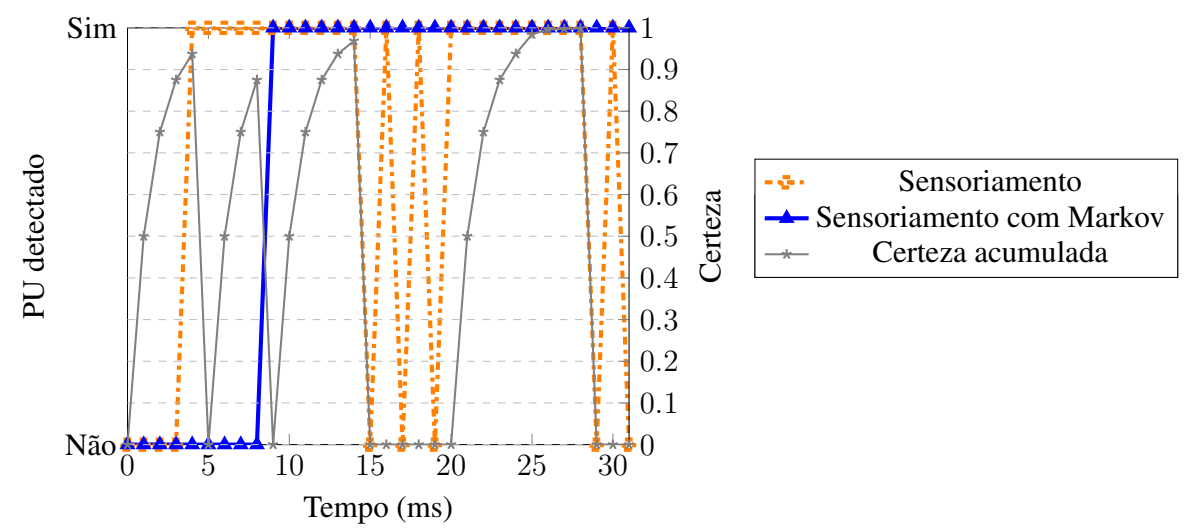

Figura 3. Suavização dos resultados do sensoriamento com a cadeia de Markov

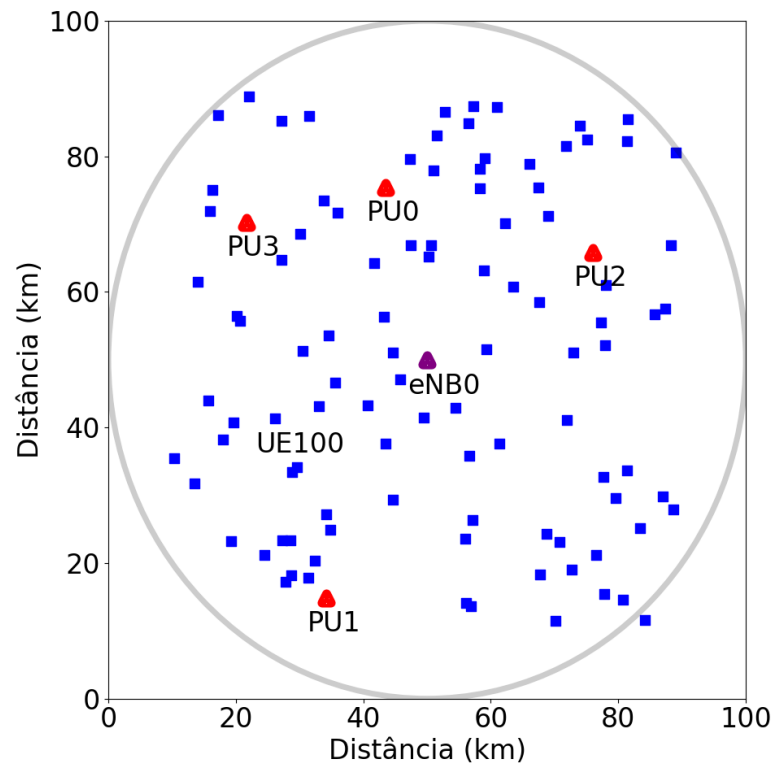

(a) Topologia de célula com 100 UEs e de raio de $50 \mathrm{Km}$

\begin{tabular}{|c|c|c|}
\hline Dispositivo & $\begin{array}{c}\text { Potência de } \\
\text { Transmissão }\end{array}$ & $\begin{array}{c}\text { Ganho da } \\
\text { Antena }\end{array}$ \\
\hline eNB & $53 \mathrm{dBm}$ & $9 \mathrm{dBi}$ \\
UE & $25 \mathrm{dBm}$ & $9 \mathrm{dBi}$ \\
PU & $30 \sim 40 \mathrm{dBm}$ & - \\
\hline
\end{tabular}

(b) Parâmetros de antena e potência de transmissão utilizados [5G-Range 2018]

Figura 4. Exemplo de topologia de rede e parâmetros de antena e potência de transmissão utilizados nas simulações

sentado na Figura 2(a). A probabilidade de alarmes falsos associada a curva de probabilidade é igual a $P_{f a}=0.1$, conforme modelo do canal utilizado em áreas remotas [5G-Range 2018].

\subsection{Cenários de Simulação e Métricas de Avaliação}

Os cenários de simulação utilizam uma célula que exemplifica uma área rural de grande cobertura. A célula tem $50 \mathrm{~km}$ de raio, com um eNB, quatro PUs transmitindo em diferentes canais. Foram definidos cenários com os UEs variando entre 1, 10, 20, 50 e 100, para melhor avaliar o comportamento de diferentes algoritmos de fusão ( $(O U, E$, $K$ de $N$ com $K=1 . .4$ ). PUs e UEs foram posicionados aleatoriamente na célula. Um exemplo de uma das topologias simuladas é ilustrada na Figura 4(a). Os parâmetros de potência de transmissão, ganho de antena e modelagem do canal seguem os especificados pela camada física e forma de onda em áreas rurais [5G-Range 2018], listados na Tabela 4(b). 

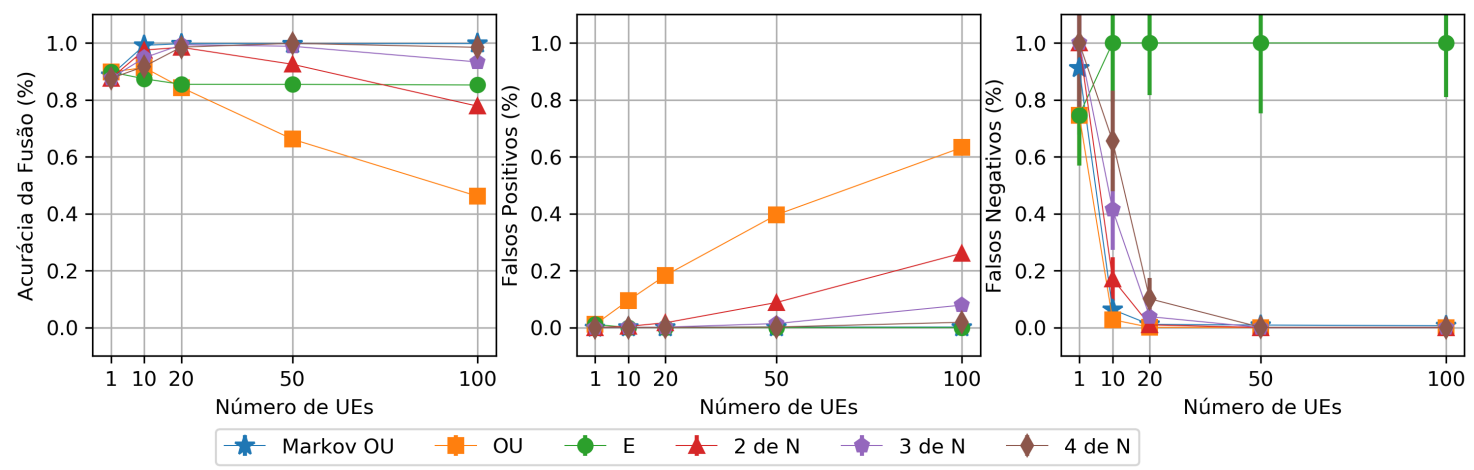

Figura 5. Resultados de simulação para diferentes algoritmos de fusão

O cenário de simulação assume que existe um canal de controle separado do canal de dados (cross-carrier scheduling), conforme o padrão 3GPP [3GPP 2019]. Como o simulador não implementa esse padrão, o canal de controle foi configurado como um canal ideal livre de erros/interferência e ao invés de usar 4 canais distintos no simulador, um único canal de $20 \mathrm{MHz}$ foi subdividido em quatro blocos de $5 \mathrm{MHz}$, cada um com um PU. O padrão de acesso dos PUs ao canal é representado pela transmissão periódica seguindo uma forma de onda quadrada com ciclo de trabalho e período aleatórios.

As métricas de avaliação para cada um dos cenários são: acurácia, $P_{f a}, P_{f n}$ e a Probabilidade de Notificar $\left(P_{\text {report }}\right)$. Estas são calculadas, respectivamente, pelas Equações 3, 4, 5, 6. $P_{\text {report }}$ indica a quantidade média de subquadros reportados por UE, que reflete a sobrecarga da transmissão dos dados de sensoriamento. Para cada combinação de fusão com e sem o mecanismo proposto, foram executadas 24 simulações com duração de 10 segundos, cada uma delas equivalentes a 10.000 amostragens por UE.

$$
\begin{gathered}
\text { acc }=\frac{\text { \#subQuadros }-\# \text { falsosNegativos }-\# \text { alarmesFalsos }}{\# \text { subQuadros }} \\
P_{f a}=\frac{\text { \#alarmesFalsos }}{\# \text { subQuadrosComPUInativo }} \\
P_{\text {fn }}=\frac{\# \text { falsosNegativos }}{\# \text { subQuadrosComPUAtivo }} \\
P_{\text {report }}=\frac{1}{\# \text { subquadros }} \sum_{t=0}^{t_{\text {final }}} P_{\text {acum }}(t)>0.9 \wedge R_{\text {Markov }}(t) \neq R_{\text {Sense }}(t)
\end{gathered}
$$

\subsection{Análise de Resultados}

Os resultados das simulações são apresentados na Tabela 1, com intervalo de confiança de $95 \%$ de acordo com o t-test. A Figura 5 ilustra os mesmos resultados, em que a primeira, segunda e terceira coluna são, respectivamente, acurácia, $P_{f a}$ e $P_{f n}$, após a fusão do sensoriamento colaborativo. Com exceção da fusão $O U$ com UEs executando o sensoriamento padrão, a qual é afetada duramente pelo crescimento exponencial dos alarmes falsos, todos os algoritmos se mostram relativamente competitivos em termos de 


\begin{tabular}{|c|c|c|c|c|c|c|c|}
\hline $\begin{array}{c}\text { Número } \\
\text { de UEs }\end{array}$ & Métrica & \multicolumn{6}{|c|}{ Fusões } \\
\hline \multirow{3}{*}{1} & Acurácia & $0.898 \pm 0.015$ & $0.875 \pm 0.021$ & $0.875 \pm 0.021$ & $0.875 \pm 0.021$ & $0.898 \pm 0.015$ & $0.886 \pm 0.022$ \\
& $P_{f a}$ & $0.011 \pm 0.001$ & $0.000 \pm 0.000$ & $0.000 \pm 0.000$ & $0.000 \pm 0.000$ & $0.011 \pm 0.001$ & $0.000 \pm 0.000$ \\
& $P_{f n}$ & $0.746 \pm 0.177$ & $1.000 \pm 0.240$ & $1.000 \pm 0.240$ & $1.000 \pm 0.240$ & $0.746 \pm 0.177$ & $0.911 \pm 0.233$ \\
\hline \multirow{3}{*}{10} & Acurácia & $0.874 \pm 0.018$ & $0.917 \pm 0.019$ & $0.948 \pm 0.016$ & $0.975 \pm 0.009$ & $0.913 \pm 0.003$ & $0.991 \pm 0.005$ \\
& $P_{f a}$ & $0.000 \pm 0.000$ & $0.000 \pm 0.000$ & $0.000 \pm 0.000$ & $0.004 \pm 0.000$ & $0.095 \pm 0.003$ & $0.001 \pm 0.000$ \\
& $P_{f n}$ & $1.000 \pm 0.207$ & $0.656 \pm 0.177$ & $0.414 \pm 0.141$ & $0.171 \pm 0.076$ & $0.027 \pm 0.017$ & $0.063 \pm 0.038$ \\
\hline \multirow{3}{*}{20} & Acurácia & $0.855 \pm 0.019$ & $0.985 \pm 0.010$ & $0.993 \pm 0.005$ & $0.984 \pm 0.001$ & $0.843 \pm 0.004$ & $0.997 \pm 0.001$ \\
& $P_{f a}$ & $0.000 \pm 0.000$ & $0.000 \pm 0.000$ & $0.001 \pm 0.000$ & $0.017 \pm 0.001$ & $0.183 \pm 0.006$ & $0.001 \pm 0.000$ \\
& $P_{f n}$ & $1.000 \pm 0.184$ & $0.101 \pm 0.073$ & $0.038 \pm 0.034$ & $0.009 \pm 0.010$ & $0.001 \pm 0.001$ & $0.011 \pm 0.004$ \\
\hline \multirow{3}{*}{50} & Acurácia & $0.854 \pm 0.025$ & $0.998 \pm 0.000$ & $0.988 \pm 0.001$ & $0.925 \pm 0.002$ & $0.662 \pm 0.010$ & $0.997 \pm 0.000$ \\
& $P_{f a}$ & $0.000 \pm 0.000$ & $0.002 \pm 0.000$ & $0.014 \pm 0.001$ & $0.088 \pm 0.004$ & $0.396 \pm 0.016$ & $0.002 \pm 0.000$ \\
& $P_{f n}$ & $1.000 \pm 0.247$ & $0.001 \pm 0.001$ & $0.000 \pm 0.000$ & $0.000 \pm 0.000$ & $0.000 \pm 0.000$ & $0.009 \pm 0.003$ \\
\hline \multirow{3}{*}{100} & Acurácia & $0.852 \pm 0.020$ & $0.984 \pm 0.001$ & $0.933 \pm 0.002$ & $0.778 \pm 0.006$ & $0.461 \pm 0.013$ & $0.998 \pm 0.000$ \\
& $P_{f a}$ & $0.000 \pm 0.000$ & $0.018 \pm 0.001$ & $0.079 \pm 0.003$ & $0.261 \pm 0.009$ & $0.632 \pm 0.021$ & $0.002 \pm 0.000$ \\
& $P_{f n}$ & $1.000 \pm 0.190$ & $0.000 \pm 0.000$ & $0.000 \pm 0.000$ & $0.000 \pm 0.000$ & $0.000 \pm 0.000$ & $0.006 \pm 0.002$ \\
\hline
\end{tabular}

Tabela 1. Resultados das simulações

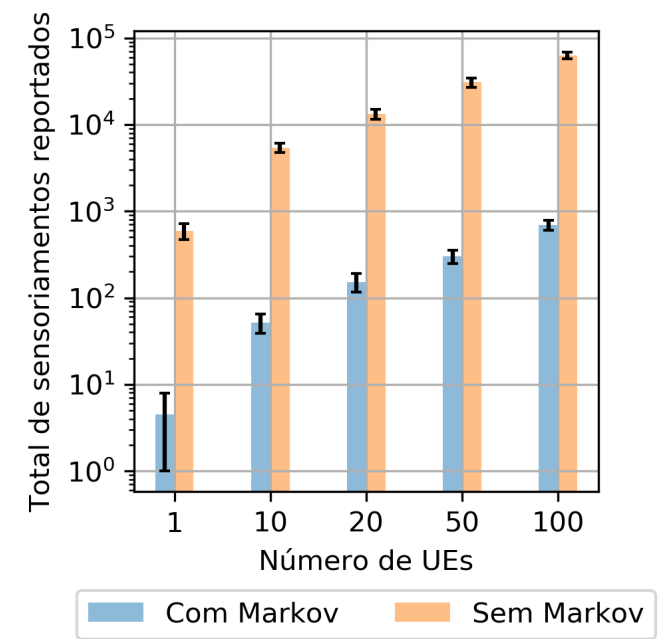

(a) Total de resultados de sensoriamento transmitidos (com $\sim 10^{3}$ subquadros simulados)

\begin{tabular}{|c|c|c|}
\hline $\begin{array}{c}\text { Número } \\
\text { de UEs }\end{array}$ & Sem Markov & Com Markov \\
\hline 1 & $0.059 \pm 0.012$ & $0.000 \pm 0.000$ \\
\hline 10 & $0.054 \pm 0.006$ & $0.001 \pm 0.000$ \\
\hline 20 & $0.066 \pm 0.009$ & $0.001 \pm 0.000$ \\
\hline 50 & $0.062 \pm 0.007$ & $0.001 \pm 0.000$ \\
\hline 100 & $0.063 \pm 0.006$ & $0.001 \pm 0.000$ \\
\hline
\end{tabular}

(b) Probabilidade de notificação de mudança de estado no canal por UE $\left(P_{\text {report }}\right)$

Figura 6. Redução do número de notificações de sensoriamentos individuais

acurácia. Apesar disto, a probabilidade de um dos nós indicar erroneamente a presença do PU cresce mais rapidamente conforme o número de UEs aumenta.

Na métrica de $P_{f a}$ observa-se o crescimento exponencial com fusões $O U$ e diversas $K d e N$, em que as últimas contam com uma menor taxa de crescimento. O efeito do mecanismo proposto é evidente ao comparar a fusão $O U$ nos dois casos, em que o número de alarmes falsos cai em até 316 vezes $(63,2 \%$ para $0,2 \%$ do total de subquadros em que o PU não estava transmitindo) para os cenários com 100 UEs. A fusão $E$ tende a apresentar uma menor $P_{f a}$ de algoritmos combinacionais, visto que exige a concordância de todos sensores indicando a presença do PU, improvável segundo a distribuição descrita pela Equação 2.

Na métrica de $P_{f a}$ é possível observar o crescimento exponencial com fusões $O U$ e diversas $K d e N$, em que as últimas contam com uma menor taxa de crescimento. O efeito do mecanismo proposto fica evidente ao comparar a fusão $O U$ com e sem o mecanismo, em que o número de alarmes falsos cai em até 316 vezes $(63,2 \%$ para $0,2 \%$ do total de subquadros em que o PU não estava transmitindo)espectro licenciado é de $P_{f n}<0.1$, o que garante um nível tolerável de colisões e interferência ao usuário do canal. A fusão 
$O U$ junto do mecanismo proposto atendeu esse critério na maioria dos cenários simulados, exceto o cenário completamente irreal com um único UE por célula e no limite superior da margem de erro no cenário com 10 UEs.

A Figura 6(a) apresenta que o número total de notificações utilizando o mecanismo proposto. Observa-se que é duas ordens de grandeza menor daquele transmitido a cada transição de estado do sensoriamento padrão. O pior caso não é apresentado, em que o sensoriamento segue a Figura 1(a), resultando em uma transmissão por subquadro por UE $\left(10^{6}\right)$ e $P_{\text {report }}=1.0$, com pior desempenho que ambos cenários ora apresentados.

Os resultados experimentais mostram que o método proposto apresenta uma redução de até $\sim 99.67 \%$ nos alarmes falsos e 98\% no tráfego de controle associado, com uma taxa de falsos negativos abaixo do limite tolerável de $10 \%$.

\section{Conclusões e Trabalhos futuros}

Este artigo propôs um novo mecanismo para filtragem de ruído no sensoriamento individual em uma rede 5G para áreas remotas com base em cadeias de Markov. Os resultados experimentais mostram que o mecanismo proposto apresentou ser eficiente mesmo em cenários com grande números de dispositivos. O método permite que a detecção possa diminuir os alarmes falsos sem aumento significativo dos falsos negativos. O método também diminui o número total de notificações em duas ordens de grandeza daquele observado a cada transição de estado do sensoriamento padrão.

Como trabalhos futuros, planeja-se ampliar o mecanismo para incluir a funcionalidade de mitigação do impacto que UEs maliciosos podem ter no sensoriamento colaborativo ao transmitir valores fraudulentos de sensoriamento individual.

\section{Agradecimentos}

Este trabalho foi realizado com suporte do projeto 5G-Range (www.5g-range.eu), aprovado na 4a. Chamada EU-BR em TICs, financiada pelo CTIC/RNP/MCTIC.

\section{Referências}

3GPP (2009). 3GPP TS 21.101 - V6.10.0 - Technical Specifications and Technical Reports for a UTRAN-based 3GPP system (Release 6). TS 21.101.

3GPP (2019). 3GPP TS 36.300 - V15.7.0 - Technical Specification Group Radio Access Network; Evolved Universal Terrestrial Radio Access (E-UTRA) and Evolved Universal Terrestrial Radio Access Network (E-UTRAN); Overall description; Stage 2(Release 15) . TS 36.300.

5G-Range (2018). Spectrum sensing to complement databases. Technical report. http://5g-range.eu/wp-content/uploads/2018/04/D4 . 2-Spectrum-Sensing-to-Complement-Databases.pdf.

Akyildiz, I. F., Lo, B. F., and Balakrishnan, R. (2011). Cooperative spectrum sensing in cognitive radio networks: A survey. Physical Communication, 4(1):40-62.

Alvi, S. A., Younis, M. S., Imran, M., and e Amin, F. (2014). A weighted linear combining scheme for cooperative spectrum sensing. Procedia Computer Science, 32:149-157. 
Baldo, N., Miozzo, M., Requena-Esteso, M., and Nin-Guerrero, J. (2011). An open source product-oriented LTE network simulator based on ns-3. In Proceedings of the 14th ACM international conference on Modeling, analysis and simulation of wireless and mobile systems - MSWiM 11. ACM Press.

Basnet, S., Jayawickrama, B. A., He, Y., Dutkiewicz, E., and Mueck, M. D. (2017). Opportunistic access to PAL channel for multi-RAT GAA transmission in spectrum access system. In 2017 IEEE 85th Vehicular Technology Conference (VTC Spring). IEEE.

Beckman, R., Channakeshava, K., Huang, F., Vullikanti, V. S. A., Marathe, A., Marathe, M. V., and Pei, G. (2010). Implications of dynamic spectrum access on the efficiency of primary wireless market. In 2010 IEEE Symposium on New Frontiers in Dynamic Spectrum (DySPAN). IEEE.

Cichoń, K., Kliks, A., and Bogucka, H. (2016). Energy-Efficient Cooperative Spectrum Sensing: A Survey. IEEE Communications Surveys \& Tutorials, 18:1861 - 1886.

Ferreira, G., Barreto, P. S., Caetano, M., Alchieri, E., Vartiainen, J., Karvonen, H., Matinmikko-Blue, M., and Seki, J. (2019). A tool for developing collaborative sensing and cognitive mac layer solutions for $5 \mathrm{~g}$ in rural areas. In 6th International Symposium on Wireless Communication Systems (ISWCS). IEEE.

Foukalas, F., Karetsos, G. T., and Chatzimisios, P. (2013). Cross-layer design of csma/ca with spectrum sensing for cognitive radio networks. In ISWCS 2013; The Tenth International Symposium on Wireless Communication Systems, pages 1-5.

Fu, Y., Yang, F., and He, Z. (2018). A quantization-based multibit data fusion scheme for cooperative spectrum sensing in cognitive radio networks. Sensors, 18(2):473.

Hu, F., Chen, B., and Zhu, K. (2018). Full spectrum sharing in cognitive radio networks toward 5g: A survey. IEEE Access, 6:15754-15776.

Khan, Z., Lehtomäki, J. J., Mustonen, M., and Matinmikko, M. (2011). Sensing order dispersion for autonomous cognitive radios. In Proceedings of the 6th International ICST Conference on Cognitive Radio Oriented Wireless Networks and Communications. IEEE.

Li, X., Petrova, M., and Mahonen, P. (2012). FCSS: CSMA/CA based fast cooperative spectrum sensing over multiband cognitive networks. In 2012 IEEE 23rd International Symposium on Personal, Indoor and Mobile Radio Communications - (PIMRC). IEEE.

Liu, J., Yang, L., Zhang, P., Zhang, Z., and Zheng, Y. (2014). Low-overhead cooperative spectrum sensing technology for cognitive radio networks. In International Conference on Cyberspace Technology (CCT 2014). Institution of Engineering and Technology.

Malady, A. C. and da Silva, C. R. C. M. (2008). Clustering methods for distributed spectrum sensing in cognitive radio systems. In MILCOM 2008 - 2008 IEEE Military Communications Conference. IEEE.

Maternia, M., El Ayoubi, S. E., Fallgren, M., Spapis, P., Qi, Y., Martín-Sacristán, D., Carrasco, , Fresia, M., Payaró, M., Schubert, M., Bedo, J. S., and Kulkarni, V. (2016). 5G PPP Use Cases and Performance Evaluation Models. 5GPPP. 
Pirinen, P., Saarnisaari, H., van de Beek, J., Matinmikko-Blue, M., Nilsson, R., and Latvaaho, M. (2019). Wireless connectivity for remote and arctic areas - food for thought. In 6th International Symposium on Wireless Communication Systems (ISWCS). IEEE.

Saarnisaari, H. and Vartiainen, J. (2018). Spectrum window based signal detection at low SNR. In 2018 International Conference on Military Communications and Information Systems (ICMCIS). IEEE.

Shafiq, M., Ahmad, M., Afzal, M. K., Ali, A., Irshad, A., and Choi, J.-G. (2019). Handshake sense multiple access control for cognitive radio-based IoT networks. Sensors, 19(2):241.

Sum, C., L. L. Z. M. K. F. . H. H. (2013). Design Considerations of IEEE 802.15.4m Low-Rate WPAN in TV white space. IEEE Communications Magazine, 51:74-82.

Tan, L. T. and Le, L. B. (2014). Joint cooperative spectrum sensing and MAC protocol design for multi-channel cognitive radio networks. EURASIP Journal on Wireless Communications and Networking, 2014(1).

Teguig, D., Scheers, B., and Le Nir, V. (2012). Data fusion schemes for cooperative spectrum sensing in cognitive radio networks. In 2012 Military Communications and Information Systems Conference (MCC), pages 1-7. IEEE.

Union, I. T. (2015). Radiocom sector (itu-r) final acts - world radiocommunication conference (wrc-15). Technical report. https://www.itu.int/dms_pub/itu-r/ opb/act/R-ACT-WRC.12-2015-PDF-E.pdf.

Valenta, V., Fedra, Z., Marsalek, R., Baudoin, G., and Villegas, M. (2009). Towards cognitive radio networks: Spectrum utilization measurements in suburb environment. In 2009 IEEE Radio and Wireless Symposium. IEEE.

Vartiainen, J., Matinmikko-Blue, M., Karvonen, H., Mendes, L., Matos, A., and Silva, C. (2019). Performance of WIBA energy detector in rural and remote area channel. In 2019 16th International Symposium on Wireless Communication Systems (ISWCS). IEEE.

Wang, D., Song, B., Chen, D., and Du, X. (2019). Intelligent Cognitive Radio in 5G: AI-Based Hierarchical Cognitive Cellular Networks. IEEE Wireless Communications, $26: 54-61$.

Wang, S., Liu, H., and Liu, K. (2015). An improved clustering cooperative spectrum sensing algorithm based on modified double-threshold energy detection and its optimization in cognitive wireless sensor networks. International Journal of Distributed Sensor Networks, 2015:1-7.

Wang, X. Y., Wong, A., and Ho, P.-H. (2010). Extended knowledge-based reasoning approach to spectrum sensing for cognitive radio. IEEE Transactions on Mobile Computing, 9(4):465-478.

Yau, K.-L. A., Qadir, J., Wu, C., Imran, M. A., and Ling, M. H. (2018). CognitionInspired 5G Cellular Networks: A Review and the Road Ahead. IEEE Access, 6:35072 -35090 .

Yucek, T. and Arslan, H. (2009). A survey of spectrum sensing algorithms for cognitive radio applications. IEEE Communications Surveys \& Tutorials, 11(1):116-130. 